Korean J. Math. 19 (2011), No. 4, pp. 465-480

\title{
IDENTITIES ABOUT INFINITE SERIES CONTAINING HYPERBOLIC FUNCTIONS AND TRIGONOMETRIC FUNCTIONS
}

\author{
Sung-GEun Lim
}

\begin{abstract}
B. C. Berndt established many identities about infinite series. In this paper, continuing his work, we find new identities about infinite series containing hyperbolic functions and trigonometric functions.
\end{abstract}

\section{Introduction and preliminaries}

B. C. Berndt $[2,3]$ found a lot of identities about infinite series using a certain modular transformation formula that originally stems from the generalized Eisenstein series. It seems that all his findings on infinite series look like those found in the Notebooks of Ramanujan [6]. In fact, some of Berndt's results are stated in the Notebooks and others are generalizations of formulas of Ramanujan. Recently he gave a suggestion that analogous results of his work could be found from the modular transformation formula in [3]. Following his suggestion, the author derived a lot of new series relation between infinite series $[4,5]$. In this paper, we find more of new series relations between infinite series, some of which are compared with series relations in $[2,3,4]$. For example, we find that, for $k<-1$,

$$
\sum_{n=0}^{\infty} \frac{(-1)^{n} \operatorname{csch}((2 n+1) \pi / 2)}{(2 n+1)^{2 k+2}}=(-1)^{k+1} 2^{-2 k-2} \sum_{n=1}^{\infty} \frac{(-1)^{n} \operatorname{sech}(n \pi)}{n^{2 k+2}}
$$

Received November 5, 2011. Revised December 12, 2011. Accepted December 14, 2011.

2000 Mathematics Subject Classification: 34A25, 11M36.

Key words and phrases: infinite series, modular transformation, Eisenstein series. 
and, for $\alpha, \beta>0$ with $\alpha \beta=\pi^{2}$,

$$
\begin{aligned}
\alpha^{1 / 2} \sum_{n=0}^{\infty} \operatorname{sech}((\alpha-\pi i)(2 n+1) /(4 c)) \\
=(-\beta)^{1 / 2} \sum_{n=0}^{\infty} \operatorname{sech}((\beta+\pi i)(2 n+1) /(4 c))
\end{aligned}
$$

where $c$ is a positive integer(See Corollary 2.9 and Corollary 2.19).

In this paper, we use the following notations. Let $e(w)=e^{2 \pi i w}$. We choose the branch of the argument for a complex $w$ with $-\pi \leq$ $\arg w<\pi . V \tau=V(\tau)=(a \tau+b) /(c \tau+d)$ always denote a modular transformation with $c>0$ for every complex $\tau$. Let $r=\left(r_{1}, r_{2}\right)$ and $h=\left(h_{1}, h_{2}\right)$ denote real vectors, and the associated vectors $R$ and $H$ are defined by $R=\left(R_{1}, R_{2}\right)=\left(a r_{1}+c r_{2}, b r_{1}+d r_{2}\right)$ and $H=\left(H_{1}, H_{2}\right)=$ $\left(d h_{1}-b h_{2},-c h_{1}+a h_{2}\right)$. Let $\lambda$ denote the characteristic function of the integers. For a real number $x,[x]$ denotes the greatest integer less than or equal to $x$ and $\{x\}:=x-[x]$. For real $\alpha, x$ and $\operatorname{Re}(s)>1$, let

$$
\psi(x, \alpha, s):=\sum_{n+\alpha>0} \frac{e(n x)}{(n+\alpha)^{s}} .
$$

If $x$ is an integer and $\alpha$ is not an integer, then $\psi(x, \alpha, s)=\zeta(s,\{\alpha\})$, where $\zeta(s, x)$ is the Hurwitz zeta-function. The function $\psi(x, \alpha, s)$ can be analytically continued to the entire $s$-plane [1] except for a possible simple pole at $s=1$ when $x$ is an integer. Let $\mathbb{H}=\{\tau \in \mathbb{C} \mid \operatorname{Im}(\tau)>0\}$, the upper half-plane. For $\tau \in \mathbb{H}$ and an arbitrary complex numbers $s$, define

$$
A(\tau, s ; r, h):=\sum_{m+r_{1}>0} \sum_{n-h_{2}>0} \frac{e\left(m h_{1}+\left(\left(m+r_{1}\right) \tau+r_{2}\right)\left(n-h_{2}\right)\right)}{\left(n-h_{2}\right)^{1-s}} .
$$

Let

$$
H(\tau, s ; r, h):=A(\tau, s ; r, h)+e(s / 2) A(\tau, s ;-r,-h) .
$$

We now state the theorem which is important for our results.

Theorem 1.1. [2]. Let $Q=\{\tau \in \mathbb{C} \mid \operatorname{Re}(\tau)>-d / c\}$ and $\varrho=c\left\{R_{2}\right\}-$ $d\left\{R_{1}\right\}$. Then for $\tau \in Q$ and all $s$,

$$
\begin{aligned}
& (c \tau+d)^{-s} H(V \tau, s ; r, h)=H(\tau, s ; R, H) \\
& -\lambda\left(r_{1}\right) e\left(-r_{1} h_{1}\right)(c \tau+d)^{-s} \Gamma(s)(-2 \pi i)^{-s}\left(\psi\left(h_{2}, r_{2}, s\right)+e(s / 2) \psi\left(-h_{2},-r_{2}, s\right)\right) \\
& +\lambda\left(R_{1}\right) e\left(-R_{1} H_{1}\right) \Gamma(s)(-2 \pi i)^{-s}\left(\psi\left(H_{2}, R_{2}, s\right)+e(-s / 2) \psi\left(-H_{2},-R_{2}, s\right)\right) \\
& +(2 \pi i)^{-s} L(\tau, s ; R, H),
\end{aligned}
$$


where

$$
\begin{aligned}
& L(\tau, s ; R, H) \\
& :=\sum_{j=1}^{c^{\prime}} e\left(-H_{1}\left(j+\left[R_{1}\right]-c\right)-H_{2}\left(\left[R_{2}\right]+1+[(j d+\varrho) / c]-d\right)\right) \\
& \quad \cdot \int_{C} u^{s-1} \frac{e^{-(c \tau+d)\left(j-\left\{R_{1}\right\}\right) u / c}}{e^{-(c \tau+d) u}-e\left(c H_{1}+d H_{2}\right)} \frac{e^{\{(j d+\varrho) / c\} u}}{e^{u}-e\left(-H_{2}\right)} d u,
\end{aligned}
$$

where $C$ is a loop beginning at $+\infty$, proceeding in the upper half-plane, encircling the origin in the positive direction so that $u=0$ is the only zero of

$$
\left(e^{-(c \tau+d) u}-e\left(c H_{1}+d H_{2}\right)\right)\left(e^{u}-e\left(-H_{2}\right)\right)
$$

lying "inside" the loop, and then returning to $+\infty$ in the lower half plane. Here, we choose the branch of $u^{s}$ with $0<\arg u<2 \pi$.

Remark 1.2. Theorem 1.1 is true for $\tau \in Q$. But, after the evaluation of $L(\tau, s ; R, H)$ for an integer $s$, it will be valid for all $\tau \in \mathbb{H}$ by analytic continuation.

We shall use two polynomials. One is the Bernoulli polynomials $B_{n}(x), n \geq 0$, defined by

$$
\frac{t e^{x t}}{e^{t}-1}=\sum_{n=0}^{\infty} B_{n}(x) \frac{t^{n}}{n !}(|t|<2 \pi) .
$$

The $n$-th Bernoulli number $B_{n}, n \geq 0$, is defined by $B_{n}=B_{n}(0)$. Put $\bar{B}_{n}(x)=B_{n}(\{x\}), n \geq 0$. The other is the Euler polynomials $E_{n}(x)$, $n \geq 0$, defined by

$$
\frac{2 e^{x t}}{e^{t}+1}=\sum_{n=0}^{\infty} E_{n}(x) \frac{t^{n}}{n !}(|t|<\pi) .
$$

The Euler numbers $E_{n}$ are defined by

$$
E_{n}:=2^{n} E_{n}\left(\frac{1}{2}\right), n \geq 0 .
$$

Put $\bar{E}_{n}(x)=E_{n}(\{x\}), n \geq 0$. 


\section{Infinite series identities}

From now on, we let $V$ be a modular transformation corresponding to

$$
\left(\begin{array}{cc}
1 & -1 \\
c & 1-c
\end{array}\right)
$$

for $c>0$. Put $r=\left(r_{1}, r_{2} / c\right)$. Then

$$
R_{1}=r_{1}+r_{2}, R_{2}=-r_{1}-r_{2}+\frac{r_{2}}{c} .
$$

Replacing $c \tau+1-c$ by $z$, we have

$$
V \tau=\frac{1}{c}-\frac{1}{c z}, \tau=1-\frac{1}{c}+\frac{1}{c} z .
$$

If $\tau \in Q$, then $\operatorname{Re} z>0$ and $z \in \mathbb{H}$. By Remark 1.2, we shall put $z=\pi i / \alpha$ for a positive real number $\alpha$. In this section, we consider three cases of $h=\left(h_{1}, h_{2}\right)$, i.e., $h=(1 / 2,1 / 2),(1 / 2,0)$ and $(0,1 / 2)$. We also suppose that $r_{1}$ and $r_{2}$ are not integers. In this case, $\lambda\left(r_{1}\right)=\lambda\left(R_{1}\right)=0$. By Theorem 1.1, for any integer $m$ and $z \in \mathbb{H}$ with $\operatorname{Re} z>0$,

$$
z^{m} H(V \tau,-m ; r, h)=H(\tau,-m ; R, H)+(2 \pi i)^{m} L(\tau,-m ; R, H) .
$$

For $r_{1}$ not an integer,

$$
\begin{array}{r}
H(V \tau, s ; r, h)=e\left(-\left[r_{1}\right] h_{1}\right) \sum_{n-h_{2}>0} \frac{e\left(\left(\left\{r_{1}\right\} V \tau+r_{2} / c\right)\left(n-h_{2}\right)\right)}{\left(n-h_{2}\right)^{1-s}\left(1-e\left(h_{1}+V \tau\left(n-h_{2}\right)\right)\right)} \\
+e^{\pi i s} e\left(-\left(\left[r_{1}\right]+1\right) h_{1}\right) \sum_{n+h_{2}>0} \frac{e\left(\left(\left(\left(1-\left\{r_{1}\right\}\right) V \tau-r_{2} / c\right)\left(n+h_{2}\right)\right)\right.}{\left(n+h_{2}\right)^{1-s}\left(1-e\left(-h_{1}+V \tau\left(n+h_{2}\right)\right)\right)},
\end{array}
$$

and, for $R_{1}$ not an integer,

$$
\begin{gathered}
H(\tau, s ; R, H)=e\left(-\left[R_{1}\right] H_{1}\right) \sum_{n-H_{2}>0} \frac{e\left(\left(\left\{R_{1}\right\} \tau+R_{2}\right)\left(n-H_{2}\right)\right)}{\left(n-H_{2}\right)^{1-s}\left(1-e\left(H_{1}+\tau\left(n-H_{2}\right)\right)\right)} \\
+e^{\pi i s} e\left(-\left(\left[R_{1}\right]+1\right) H_{1}\right) \sum_{n+H_{2}>0} \frac{e\left(\left(\left(1-\left\{R_{1}\right\}\right) \tau-R_{2}\right)\left(n+H_{2}\right)\right)}{\left(n+H_{2}\right)^{1-s}\left(1-e\left(-H_{1}+\tau\left(n+H_{2}\right)\right)\right)} .
\end{gathered}
$$

Theorem 2.1. Let $\alpha, \beta>0$ with $\alpha \beta=\pi^{2}$. Let $r_{1}$ and $r_{2}$ be real numbers such that $r_{1}$ and $r_{1}+r_{2}$ are not integers. Then, for any integer $k$ and for any positive even integer $c$,

$$
\begin{aligned}
& (-1)^{\left[r_{1}\right]} \alpha^{-k} \sum_{n=0}^{\infty} \frac{\sinh \left(\left(\left(2\left\{r_{1}\right\}-1\right)(\alpha-\pi i)-2 \pi i r_{2}\right)(2 n+1) /(2 c)\right)}{(2 n+1)^{2 k+1} \cosh ((\alpha-\pi i)(2 n+1) /(2 c))} \\
& =(-1)^{\left[r_{1}+r_{2}\right]}(-\beta)^{-k} \sum_{n=0}^{\infty} \frac{\sinh \left(\left(\left(2\left\{r_{1}+r_{2}\right\}-1\right)(\beta+\pi i)-2 \pi i r_{2}\right)(2 n+1) /(2 c)\right)}{(2 n+1)^{2 k+1} \cosh ((\beta+\pi i)(2 n+1) /(2 c))}
\end{aligned}
$$




$$
\begin{aligned}
+\frac{(-1)^{\left[r_{1}+r_{2}\right]}}{4} \sum_{j=1}^{c}(-1)^{j+\left[\left(j+r_{2}-\left\{r_{1}+r_{2}\right\}\right) / c\right]} \sum_{\ell=0}^{2 k} \frac{E_{\ell}\left(\left(j-\left\{r_{1}+r_{2}\right\}\right) / c\right)}{\ell !} \\
\cdot \frac{\bar{E}_{2 k-\ell}\left(\left(j+r_{2}-\left\{r_{1}+r_{2}\right\}\right) / c\right)}{(2 k-\ell) !}(-\pi i)^{\ell+1} \alpha^{k-\ell},
\end{aligned}
$$

and, for any positive odd integer $c$,

$$
\begin{gathered}
(-1)^{\left[r_{1}\right]} \alpha^{-k} \sum_{n=0}^{\infty} \frac{\sinh \left(\left(\left(2\left\{r_{1}\right\}-1\right)(\alpha-\pi i)-2 \pi i r_{2}\right)(2 n+1) /(2 c)\right)}{(2 n+1)^{2 k+1} \cosh ((\alpha-\pi i)(2 n+1) /(2 c))} \\
=\frac{(-1)^{\left[r_{1}+r_{2}\right]}}{2^{2 k+1}}(-\beta)^{-k} \sum_{n=1}^{\infty} \frac{\sinh \left(\left(\left(2\left\{r_{1}+r_{2}\right\}-1\right)(\beta+\pi i)-2 \pi i r_{2}\right) n / c\right)}{n^{2 k+1} \cosh ((\beta+\pi i) n / c)} \\
+\frac{(-1)^{\left[r_{1}+r_{2}\right]}}{2} \sum_{j=1}^{c}(-1)^{j+1} \sum_{\ell=0}^{2 k+1} \frac{E_{\ell}\left(\left(j-\left\{r_{1}+r_{2}\right\}\right) / c\right)}{\ell !} \\
\cdot \frac{\bar{B}_{2 k+1-\ell}\left(\left(j+r_{2}-\left\{r_{1}+r_{2}\right\}\right) / c\right)}{(2 k+1-\ell) !}(-\pi i)^{\ell+1} \alpha^{k-\ell} .
\end{gathered}
$$

Proof. Let $h=(1 / 2,1 / 2)$ and $m=2 k$ in $(2.1)$. Then we have from (2.2) that

$$
\begin{aligned}
H(V \tau,-2 k ; r, h) & \\
= & (-1)^{\left[r_{1}\right]} 2^{2 k+1} \sum_{n=1}^{\infty} \frac{e\left(\left(\left\{r_{1}\right\}(1-1 / z)+r_{2}\right)(2 n-1) /(2 c)\right)}{(2 n-1)^{2 k+1}(1+e((1-1 / z)(2 n-1) /(2 c)))} \\
& -(-1)^{\left[r_{1}\right]} 2^{2 k+1} \sum_{n=1}^{\infty} \frac{e\left(\left(\left(1-\left\{r_{1}\right\}\right)(1-1 / z)-r_{2}\right)(2 n-1) /(2 c)\right)}{(2 n-1)^{2 k+1}(1+e((1-1 / z)(2 n-1) /(2 c)))} \\
= & (-1)^{\left[r_{1}\right]} 2^{2 k+1} \sum_{n=1}^{\infty} \frac{\sinh \left(\pi i\left(\left(2\left\{r_{1}\right\}-1\right)(1-1 / z)+2 r_{2}\right)(2 n-1) /(2 c)\right)}{(2 n-1)^{2 k+1} \cosh (\pi i(1-1 / z)(2 n-1) /(2 c))} .
\end{aligned}
$$

If $c$ is even, then $\left\{H_{1}\right\}=0$ and $\left\{H_{2}\right\}=1 / 2$. Thus, for $c$ even, it follows from (2.3) that

$$
\begin{aligned}
& H(\tau,-2 k ; R, H) \\
= & 2^{2 k+1} \sum_{n=0}^{\infty} \frac{e^{\pi i\left(\left\{R_{1}\right\} \tau+R_{2}\right)(2 n+1)}+e^{-\pi i\left(\left\{R_{1}\right\} \tau+R_{2}\right)(2 n+1)} e^{\pi i \tau(2 n+1)}}{(2 n+1)^{2 k+1}\left(1-e^{\pi i \tau(2 n+1)}\right)} \\
(2.5)= & (-1)^{\left[r_{1}+r_{2}\right]} 2^{2 k+1} \sum_{n=0}^{\infty} \frac{\sinh \left(\pi i\left(\left(2\left\{r_{1}+r_{2}\right\}-1\right)(z-1)+2 r_{2}\right)(2 n+1) /(2 c)\right)}{(2 n+1)^{2 k+1} \cosh (\pi i(z-1)(2 n+1) /(2 c))} .
\end{aligned}
$$

If $c$ is odd, then $\left\{H_{1}\right\}=1 / 2$ and $\left\{H_{2}\right\}=0$. So, for $c$ odd, (2.3) gives

$$
\begin{aligned}
& H(\tau,-2 k ; R, H) \\
& =(-1)^{\left[R_{1}\right]} \sum_{n=1}^{\infty} \frac{\left.\left.e\left(\left(\left\{R_{1}\right\} \tau+R_{2}\right) n\right)\right)-e\left(-\left(\left\{R_{1}\right\} \tau+R_{2}\right) n\right)\right) e(\tau n)}{n^{2 k+1}(1+e(\tau n))}
\end{aligned}
$$


$(2.6)=(-1)^{\left[r_{1}+r_{2}\right]} \sum_{n=1}^{\infty} \frac{\sinh \left(\pi i\left(\left(2\left\{r_{1}+r_{2}\right\}-1\right)(z-1)+2 r_{2}\right) n / c\right)}{n^{2 k+1} \cosh (\pi i(z-1) n / c)}$.

We see that

$$
\begin{aligned}
& \frac{e^{-z u\left(j-\left\{R_{1}\right\}\right) / c}}{e^{-z u}+1}=\frac{1}{2} \sum_{n=0}^{\infty} E_{n}\left(\frac{j-\left\{R_{1}\right\}}{c}\right) \frac{(-z u)^{n}}{n !}, \\
& \frac{e^{\{(j(1-c)+\varrho) / c\} u}}{e^{u}+1}=\frac{1}{2} \sum_{n=0}^{\infty} \bar{E}_{n}\left(\frac{j+\varrho}{c}\right) \frac{u^{n}}{n !}, \\
& \frac{e^{\{(j(1-c)+\varrho) / c\} u}}{e^{u}-1}=u^{-1} \sum_{n=0}^{\infty} \bar{B}_{n}\left(\frac{j+\varrho}{c}\right) \frac{u^{n}}{n !},
\end{aligned}
$$

and

$$
\left[\frac{j(1-c)+\varrho}{c}\right]=-j-\left[R_{1}\right]-\left[R_{2}\right]+\left[\frac{j+r_{2}-\left\{R_{1}\right\}}{c}\right] .
$$

Then, in case of $c$ even, we have that

$$
\begin{aligned}
& L(\tau,-2 k ; R, H) \\
& =\frac{1}{4} \sum_{j=1}^{c} e\left(-\frac{1}{2}\left(\left[R_{2}\right]+c+\left[\frac{j(1-c)+\varrho}{c}\right]\right)\right) \\
& \cdot \int_{C} u^{-2 k-1} \sum_{n=0}^{\infty} E_{n}\left(\frac{j-\left\{R_{1}\right\}}{c}\right) \frac{(-z u)^{n}}{n !} \sum_{m=0}^{\infty} \bar{E}_{m}\left(\frac{j+\varrho}{c}\right) \frac{u^{m}}{m !} d u \\
& =\frac{(-1)^{\left[r_{1}+r_{2}\right]}}{2} \pi i \sum_{j=1}^{c}(-1)^{j+\left[\left(j+r_{2}-\left\{r_{1}+r_{2}\right\}\right) / c\right]} \sum_{\ell=0}^{2 k} \frac{E_{\ell}\left(\left(j-\left\{r_{1}+r_{2}\right\}\right) / c\right)}{\ell !} \\
& \cdot \frac{\bar{E}_{2 k-\ell}\left(\left(j+r_{2}-\left\{r_{1}+r_{2}\right\}\right) / c\right)}{(2 k-\ell) !}(-z)^{\ell}
\end{aligned}
$$

and, in case of $c$ odd,

$$
\begin{aligned}
& L(\tau,-2 k ; R, H) \\
& =\frac{1}{2} \sum_{j=1}^{c} e\left(-\frac{1}{2}\left(j+\left[R_{1}\right]-c\right)\right) \\
& \quad \cdot \int_{C} u^{-2 k-2} \sum_{n=0}^{\infty} E_{n}\left(\frac{j-\left\{R_{1}\right\}}{c}\right) \frac{(-z u)^{n}}{n !} \sum_{m=0}^{\infty} \bar{B}_{m}\left(\frac{j+\varrho}{c}\right) \frac{u^{m}}{m !} d u \\
& =(-1)^{\left[r_{1}+r_{2}\right]+1} \pi i \sum_{j=1}^{c}(-1)^{j} \sum_{\ell=0}^{2 k+1} \frac{E_{\ell}\left(\left(j-\left\{r_{1}+r_{2}\right\}\right) / c\right)}{\ell !} \\
& \cdot \frac{\bar{B}_{2 k+1-\ell}\left(\left(j+r_{2}-\left\{r_{1}+r_{2}\right\}\right) / c\right)}{(2 k+1-\ell) !}(-z)^{\ell} .
\end{aligned}
$$


Now, plugging (2.4), (2.5), (2.6), (2.7) and (2.8) into (2.1) and letting $z=\pi i / \alpha$, we prove the theorem.

Corollary 2.2. Let $r_{1}$ be a real number with $0<r_{1}<1$. Then

$$
\begin{aligned}
\alpha^{-k} \sum_{n=0}^{\infty} & \frac{\cosh \left((2 n+1)\left(2 r_{1}-1\right) \alpha / 2\right) \cos \left((2 n+1) \pi r_{1}\right)}{(2 n+1)^{2 k+1} \sinh ((2 n+1) \alpha / 2)} \\
=- & 2^{-2 k-1}(-\beta)^{-k} \sum_{n=1}^{\infty} \frac{\sinh \left(\left(2 r_{1}-1\right) n \beta\right) \cos \left(2 \pi n r_{1}\right)}{n^{2 k+1} \cosh (n \beta)} \\
& -\frac{1}{2} \sum_{\ell=0}^{k} \frac{E_{2 \ell+1}\left(1-r_{1}\right) B_{2 k-2 \ell}\left(1-r_{1}\right)}{(2 \ell+1) !(2 k-2 \ell) !} \alpha^{k-\ell+1}(-\beta)^{\ell+1} .
\end{aligned}
$$

Proof. Put $c=1, r_{2}=0$ and let $0<r_{1}<1$ in Theorem 2.1 and equate the real parts.

Corollary 2.3. Let $r_{1}$ be a real number with $0<r_{1}<1$. Then

$$
\begin{aligned}
\alpha^{-k} & \sum_{n=0}^{\infty} \frac{\sinh \left((2 n+1)\left(2 r_{1}-1\right) \alpha / 2\right) \sin \left((2 n+1) \pi r_{1}\right)}{(2 n+1)^{2 k+1} \sinh ((2 n+1) \alpha / 2)} \\
= & 2^{-2 k-1}(-\beta)^{-k} \sum_{n=1}^{\infty} \frac{\cosh \left(\left(2 r_{1}-1\right) n \beta\right) \sin \left(2 \pi n r_{1}\right)}{n^{2 k+1} \cosh (n \beta)} \\
& -\frac{\pi}{2} \sum_{\ell=0}^{k} \frac{E_{2 \ell}\left(1-r_{1}\right) B_{2 k+1-2 \ell}\left(1-r_{1}\right)}{(2 \ell) !(2 k+1-2 \ell) !} \alpha^{k-\ell}(-\beta)^{\ell} .
\end{aligned}
$$

Proof. Put $c=1, r_{2}=0$ and let $0<r_{1}<1$ in Theorem 2.1 and equate the imaginary parts.

Theorem 2.4. Let $\alpha, \beta>0$ with $\alpha \beta=\pi^{2}$. Let $r_{1}$ and $r_{2}$ be real numbers such that $r_{1}$ and $r_{1}+r_{2}$ are not integers. Then, for any integer $k$ and for any positive even integer $c$,

$$
\begin{aligned}
(-1)^{\left[r_{1}\right]} \alpha^{-k-1 / 2} \sum_{n=0}^{\infty} \frac{\cosh \left(\left(\left(2\left\{r_{1}\right\}-1\right)(\alpha-\pi i)-2 \pi i r_{2}\right)(2 n+1) /(2 c)\right)}{(2 n+1)^{2 k+2} \cosh ((\alpha-\pi i)(2 n+1) /(2 c))} & \\
= & (-1)^{\left[r_{1}+r_{2}\right]}(-\beta)^{-k-1 / 2} \\
& \cdot \sum_{n=0}^{\infty} \frac{\cosh \left(\left(\left(2\left\{r_{1}+r_{2}\right\}-1\right)(\beta+\pi i)-2 \pi i r_{2}\right)(2 n+1) /(2 c)\right)}{(2 n+1)^{2 k+2} \cosh ((\beta+\pi i)(2 n+1) /(2 c))} \\
& -\frac{(-1)^{\left[r_{1}+r_{2}\right]}}{4} \sum_{j=1}^{c}(-1)^{j+\left[\left(j+r_{2}-\left\{r_{1}+r_{2}\right\}\right) / c\right]} \sum_{\ell=0}^{2 k+1} \frac{E_{\ell}\left(\left(j-\left\{r_{1}+r_{2}\right\}\right) / c\right)}{\ell !} \\
\cdot & \frac{\bar{E}_{2 k+1-\ell}\left(\left(j+r_{2}-\left\{r_{1}+r_{2}\right\}\right) / c\right)}{(2 k+1-\ell) !}(-\pi i)^{\ell+1} \alpha^{k-\ell+1 / 2},
\end{aligned}
$$

and, for any positive odd integer $c$,

$$
(-1)^{\left[r_{1}\right]} \alpha^{-k-1 / 2} \sum_{n=0}^{\infty} \frac{\cosh \left(\left(\left(2\left\{r_{1}\right\}-1\right)(\alpha-\pi i)-2 \pi i r_{2}\right)(2 n+1) /(2 c)\right)}{(2 n+1)^{2 k+2} \cosh ((\alpha-\pi i)(2 n+1) /(2 c))}
$$




$$
\begin{gathered}
=\frac{(-1)^{\left[r_{1}+r_{2}\right]}}{2^{2 k+2}}(-\beta)^{-k-1 / 2} \sum_{n=1}^{\infty} \frac{\cosh \left(\left(\left(2\left\{r_{1}+r_{2}\right\}-1\right)(\beta+\pi i)-2 \pi i r_{2}\right) n / c\right)}{n^{2 k+2} \cosh ((\beta+\pi i) n / c)} \\
-\frac{(-1)^{\left[r_{1}+r_{2}\right]}}{2} \sum_{j=1}^{c}(-1)^{j+1} \sum_{\ell=0}^{2 k+2} \frac{E_{\ell}\left(\left(j-\left\{r_{1}+r_{2}\right\}\right) / c\right)}{\ell !} \\
\cdot \frac{\bar{B}_{2 k+2-\ell}\left(\left(j+r_{2}-\left\{r_{1}+r_{2}\right\}\right) / c\right)}{(2 k+2-\ell) !}(-\pi i)^{\ell+1} \alpha^{k-\ell+1 / 2} .
\end{gathered}
$$

Proof. Let $h=(1 / 2,1 / 2)$ and $m=2 k+1$ in (2.1). By the same way as we derived equations (2.4), (2.5), (2.6), (2.7) and (2.8), we obtain the followings;

$$
\begin{aligned}
H(V \tau,-2 k-1 ; r, h)=(-1)^{\left[r_{1}\right]} 2^{2 k+2} \sum_{n=1}^{\infty} \frac{1}{(2 n-1)^{2 k+2}} & \\
& \cdot \frac{\cosh \left(\pi i\left(\left(2\left\{r_{1}\right\}-1\right)(1-1 / z)+2 r_{2}\right)(2 n-1) /(2 c)\right)}{\cosh (\pi i(1-1 / z)(2 n-1) /(2 c))},
\end{aligned}
$$

for $c$ even,

$$
H(\tau,-2 k-1 ; R, H)=(-1)^{\left[r_{1}+r_{2}\right]} 2^{2 k+2} \sum_{n=0}^{\infty} \frac{1}{(2 n+1)^{2 k+2}}
$$

$$
\cdot \frac{\cosh \left(\pi i\left(\left(2\left\{r_{1}+r_{2}\right\}-1\right)(z-1)+2 r_{2}\right)(2 n+1) /(2 c)\right)}{\cosh (\pi i(z-1)(2 n+1) /(2 c))},
$$

$$
\begin{aligned}
L(\tau,-2 k & -1 ; R, H)=\frac{(-1)^{\left[r_{1}+r_{2}\right]}}{2} \pi i \sum_{j=1}^{c}(-1)^{j+\left[\left(j+r_{2}-\left\{r_{1}+r_{2}\right\}\right) / c\right]} \\
& \cdot \sum_{\ell=0}^{2 k+1} \frac{E_{\ell}\left(\left(j-\left\{r_{1}+r_{2}\right\}\right) / c\right) \bar{E}_{2 k+1-\ell}\left(\left(j+r_{2}-\left\{r_{1}+r_{2}\right\}\right) / c\right)}{\ell !(2 k+1-\ell) !}(-z)^{\ell}
\end{aligned}
$$

for $c$ odd,

$$
\begin{gathered}
H(\tau,-2 k-1 ; R, H) \\
=(-1)^{\left[r_{1}+r_{2}\right]} \sum_{n=1}^{\infty} \frac{\cosh \left(\pi i\left(\left(2\left\{r_{1}+r_{2}\right\}-1\right)(z-1)+2 r_{2}\right) n / c\right)}{n^{2 k+2} \cosh (\pi i(z-1) n / c)}, \\
L(\tau,-2 k ; R, H)=(-1)^{\left[r_{1}+r_{2}\right]+1} \pi i \sum_{j=1}^{c}(-1)^{j} \sum_{\ell=0}^{2 k+2} \frac{E_{\ell}\left(\left(j-\left\{r_{1}+r_{2}\right\}\right) / c\right)}{\ell !} \\
\cdot \frac{\bar{B}_{2 k+2-\ell}\left(\left(j+r_{2}-\left\{r_{1}+r_{2}\right\}\right) / c\right)}{(2 k+2-\ell) !}(-z)^{\ell} .
\end{gathered}
$$

Now let $z=\pi i / \alpha$, put (2.9), (2.10), (2.11), (2.12) and (2.13) into (2.1), and obtain the desired results. 
Corollary 2.5. Let $r_{1}$ be a real number with $0<r_{1}<1$. Then

$$
\begin{aligned}
& \alpha^{-k-1 / 2} \sum_{n=0}^{\infty} \frac{\sinh \left((2 n+1)\left(2 r_{1}-1\right) \alpha / 2\right) \cos \left((2 n+1) \pi r_{1}\right)}{(2 n+1)^{2 k+2} \sinh ((2 n+1) \alpha / 2)} \\
& =(-1)^{k+1} 2^{-2 k-2} \beta^{-k-1 / 2} \sum_{n=1}^{\infty} \frac{\sinh \left(\left(2 r_{1}-1\right) n \beta\right) \sin \left(2 \pi n r_{1}\right)}{n^{2 k+2} \cosh (n \beta)} \\
& \quad+\frac{1}{2} \sum_{\ell=0}^{k} \frac{E_{2 \ell+1}\left(1-r_{1}\right) E_{2 k+1-2 \ell}\left(1-r_{1}\right)}{(2 \ell+1) !(2 k+1-2 \ell) !} \alpha^{k-\ell+1 / 2}(-\beta)^{\ell+1} .
\end{aligned}
$$

Proof. Put $c=1, r_{2}=0$ and let $0<r_{1}<1$ in Theorem 2.4 and equate the real parts.

Corollary 2.6. Let $r_{1}$ be a real number with $0<r_{1}<1$. Then

$$
\begin{gathered}
\alpha^{-k-1 / 2} \sum_{n=0}^{\infty} \frac{\cosh \left((2 n+1)\left(2 r_{1}-1\right) \alpha / 2\right) \sin \left((2 n+1) \pi r_{1}\right)}{(2 n+1)^{2 k+2} \sinh ((2 n+1) \alpha / 2)} \\
=(-1)^{k+1} 2^{-2 k-2} \beta^{-k-1 / 2} \sum_{n=1}^{\infty} \frac{\cosh \left(\left(2 r_{1}-1\right) n \beta\right) \cos \left(2 \pi n r_{1}\right)}{n^{2 k+2} \cosh (n \beta)} \\
+\frac{\pi}{2} \sum_{\ell=0}^{k} \frac{E_{2 \ell}\left(1-r_{1}\right) E_{2 k+2-2 \ell}\left(1-r_{1}\right)}{(2 \ell) !(2 k+2-2 \ell) !} \alpha^{k-\ell+1 / 2}(-\beta)^{\ell} .
\end{gathered}
$$

Proof. Put $c=1, r_{2}=0$ and let $0<r_{1}<1$ in Theorem 2.4 and equate the imaginary parts.

Corollary 2.7. For any positive even integer $c$,

$$
\begin{aligned}
& \alpha^{-k-1 / 2} \sum_{n=0}^{\infty} \frac{\operatorname{sech}((\alpha-\pi i)(2 n+1) /(2 c))}{(2 n+1)^{2 k+2}} \\
& =(-\beta)^{-k-1 / 2} \sum_{n=0}^{\infty} \frac{\operatorname{sech}((\beta+\pi i)(2 n+1) /(2 c))}{(2 n+1)^{2 k+2}} \\
& \quad-\frac{1}{4} \sum_{j=1}^{c}(-1)^{j} \sum_{\ell=0}^{2 k+1} \frac{E_{\ell}((j-1 / 2) / c) E_{2 k+1-\ell}((j-1 / 2) / c)}{\ell !(2 k+1-\ell) !}(-\pi i)^{\ell+1} \alpha^{k-\ell+1 / 2},
\end{aligned}
$$

and, for any positive odd integer $c$,

$$
\begin{aligned}
& \alpha^{-k-1 / 2} \sum_{n=0}^{\infty} \frac{\operatorname{sech}((\alpha-\pi i)(2 n+1) /(2 c))}{(2 n+1)^{2 k+2}} \\
& =2^{-2 k-2}(-\beta)^{-k-1 / 2} \sum_{n=1}^{\infty} \frac{\operatorname{sech}((\beta+\pi i) n / c)}{n^{2 k+2}} \\
& \quad+\frac{1}{2} \sum_{j=1}^{c}(-1)^{j} \sum_{\ell=0}^{2 k+2} \frac{E_{\ell}((j-1 / 2) / c) E_{2 k+2-\ell}((j-1 / 2) / c)}{\ell !(2 k+2-\ell) !}(-\pi i)^{\ell+1} \alpha^{k-\ell+1 / 2} .
\end{aligned}
$$

Proof. Put $r_{1}=1 / 2$ and $r_{2}=0$ in Theorem 2.4. 
COROllary 2.8 .

$$
\begin{aligned}
& \alpha^{-k-1 / 2} \sum_{n=0}^{\infty} \frac{(-1)^{n} \operatorname{csch}((2 n+1) \alpha / 2)}{(2 n+1)^{2 k+2}} \\
& =(-1)^{k+1} 2^{-2 k-2} \beta^{-k-1 / 2} \sum_{n=1}^{\infty} \frac{(-1)^{n} \operatorname{sech}(n \beta)}{n^{2 k+2}} \\
& \quad+\frac{\pi}{2} \sum_{\ell=0}^{k+1} \frac{E_{2 \ell}(1 / 2) E_{2 k+2-2 \ell}(1 / 2)}{(2 \ell) !(2 k+2-2 \ell) !} \alpha^{k-\ell+1 / 2}(-\beta)^{\ell} .
\end{aligned}
$$

Proof. Put $c=1$ in Corollary 2.7 and apply $E_{2 n+1}\left(\frac{1}{2}\right)=0, n \geq 0$.

Corollary 2.9. For $k<-1$,

$$
\sum_{n=0}^{\infty} \frac{(-1)^{n} \operatorname{csch}((2 n+1) \pi / 2)}{(2 n+1)^{2 k+2}}=(-1)^{k+1} 2^{-2 k-2} \sum_{n=1}^{\infty} \frac{(-1)^{n} \operatorname{sech}(n \pi)}{n^{2 k+2}}
$$

Proof. Put $c=1, \alpha=\beta=\pi$ in Corollary 2.7 and let $k<-1$.

COROllary 2.10 .

$$
\sum_{n=0}^{\infty}(-1)^{n} \operatorname{csch}((2 n+1) \pi / 2)=\sum_{n=1}^{\infty}(-1)^{n} \operatorname{sech}(n \pi)+\frac{1}{2}
$$

Proof. Put $c=1, k=-1$ and $\alpha=\beta=\pi$ in Corollary 2.7 .

Theorem 2.11. Let $\alpha, \beta>0$ with $\alpha \beta=\pi^{2}$. Let $r_{1}$ and $r_{2}$ be real numbers such that $r_{1}$ and $r_{1}+r_{2}$ are not integers. Then, for any integer $k$ and for any positive even integer $c$,

$$
\begin{gathered}
(-1)^{\left[r_{1}\right]} \alpha^{-k} \sum_{n=1}^{\infty} \frac{\sinh \left(\left(\left(2\left\{r_{1}\right\}-1\right)(\alpha-\pi i)-2 \pi i r_{2}\right) n / c\right)}{n^{2 k+1} \cosh ((\alpha-\pi i) n / c)} \\
=(-1)^{\left[r_{1}+r_{2}\right]}(-\beta)^{-k} \sum_{n=1}^{\infty} \frac{\sinh \left(\left(\left(2\left\{r_{1}+r_{2}\right\}-1\right)(\beta+\pi i)-2 \pi i r_{2}\right) n / c\right)}{n^{2 k+1} \cosh ((\beta+\pi i) n / c)} \\
+(-1)^{\left[r_{1}+r_{2}\right]} 2^{2 k+1} \sum_{j=1}^{c}(-1)^{j} \sum_{\ell=0}^{2 k+2} \frac{B_{\ell}\left(\left(j-\left\{r_{1}+r_{2}\right\}\right) / c\right)}{\ell !} \\
\cdot \frac{\bar{B}_{2 k+2-\ell}\left(\left(j+r_{2}-\left\{r_{1}+r_{2}\right\}\right) / c\right)}{(2 k+2-\ell) !}(-\pi i)^{\ell} \alpha^{k-\ell+1} .
\end{gathered}
$$

Proof. Let $h=(1 / 2,0)$ and $m=2 k$ in $(2.1)$. Then it follows from (2.2) that

$$
\begin{aligned}
H & (V \tau,-2 k ; r, h) \\
& =(-1)^{\left[r_{1}\right]} \sum_{n=1}^{\infty} \frac{e\left(\left(\left\{r_{1}\right\}(1-1 / z)+r_{2}\right) n / c\right)-e\left(\left(\left(1-\left\{r_{1}\right\}\right)(1-1 / z)-r_{2}\right) n / c\right)}{n^{2 k+1}(1+e((1-1 / z) n / c))} \\
(2.14) & =(-1)^{\left[r_{1}\right]} \sum_{n=1}^{\infty} \frac{\sinh \left(\pi i\left(\left(2\left\{r_{1}\right\}-1\right)(1-1 / z)+2 r_{2}\right) n / c\right)}{n^{2 k+1} \cosh (\pi i(1-1 / z) n / c)}
\end{aligned}
$$




$$
\begin{aligned}
& H(\tau,-2 k ; R, H) \\
& \quad=(-1)^{\left[r_{1}+r_{2}\right]} \sum_{n=1}^{\infty} \frac{e\left(\left(\left\{r_{1}+r_{2}\right\}(z-1)+r_{2}\right) n / c\right)-e\left(-\left(\left\{r_{1}+r_{2}\right\}(z-1)+r_{2}\right) n / c\right)}{n^{2 k+1}(1+e((z-1) n / c))} \\
& (2.15)=(-1)^{\left[r_{1}+r_{2}\right]} \sum_{n=1}^{\infty} \frac{\sinh \left(\pi i\left(\left(2\left\{r_{1}+r_{2}\right\}-1\right)(z-1)+2 r_{2}\right) n / c\right)}{n^{2 k+1} \cosh (\pi i(z-1) n / c)} .
\end{aligned}
$$

Since $c$ is even, $c H_{1}+(1-c) H_{2} \equiv H_{2} \equiv 0(\bmod 1)$. We use that

$$
\begin{aligned}
& \frac{e^{-z u\left(j-\left\{R_{1}\right\}\right) / c}}{e^{-z u}-1}=(-z u)^{-1} \sum_{m=0}^{\infty} B_{m}\left(\frac{j-\left\{R_{1}\right\}}{c}\right) \frac{(-z u)^{m}}{m !}, \\
& \frac{e^{\{(j(1-c)+\varrho) / c\} u}}{e^{u}-1}=u^{-1} \sum_{m=0}^{\infty} \bar{B}_{m}\left(\frac{j(1-c)+\varrho}{c}\right) \frac{u^{m}}{m !}
\end{aligned}
$$

and

$$
\left\{\frac{j(1-c)+\varrho}{c}\right\}=\left\{\frac{j+r_{2}-\left\{r_{1}+r_{2}\right\}}{c}\right\} .
$$

Then by the residue theorem we have

$$
\begin{aligned}
& L(\tau,-2 k ; R, H) \\
& =(-z)^{-1} \sum_{j=1}^{c} e^{-\pi i\left(j+\left[R_{1}\right]-c\right)} \int_{C} u^{-2 k-3} \sum_{m=0}^{\infty} B_{m}\left(\frac{j-\left\{R_{1}\right\}}{c}\right) \frac{(-z u)^{m}}{m !} \\
& =(-1)^{\left[r_{1}+r_{2}\right]} 2 \pi i \sum_{j=1}^{\infty}(-1)^{j} \sum_{\ell=0}^{2 k+2} \frac{B_{\ell}\left(\left(j-\left\{r_{1}+r_{2}\right\}\right) / c\right)}{\ell !} \\
& \cdot \frac{\bar{B}_{2 k+2-\ell}\left(\left(j+r_{2}-\left\{r_{1}+r_{2}\right\}\right) / c\right)}{(2 k+2-\ell) !}(-z)^{\ell-1} .
\end{aligned}
$$

Employing (2.14), (2.15) and (2.16) in (2.1) with $z=\pi i / \alpha$, we complete the proof.

For $c$ odd, if we put $h=(1 / 2,0), m=2 k$ and $z=\pi i / \alpha$ into (2.1), then we obtain the complex conjugate of the second series identity in Theorem 2.1.

Theorem 2.12. Let $\alpha, \beta>0$ with $\alpha \beta=\pi^{2}$. Let $r_{1}$ and $r_{2}$ be real numbers such that $r_{1}$ and $r_{1}+r_{2}$ are not integers. Then, for any integer $k$ and for any positive even integer $c$,

$$
(-1)^{\left[r_{1}\right]} \alpha^{-k-1 / 2} \sum_{n=1}^{\infty} \frac{\cosh \left(\left(\left(2\left\{r_{1}\right\}-1\right)(\alpha-\pi i)-2 \pi i r_{2}\right) n / c\right)}{n^{2 k+2} \cosh ((\alpha-\pi i) n / c)}
$$




$$
\begin{gathered}
=(-1)^{\left[r_{1}+r_{2}\right]}(-\beta)^{-k-1 / 2} \sum_{n=1}^{\infty} \frac{\cosh \left(\left(\left(2\left\{r_{1}+r_{2}\right\}-1\right)(\beta+\pi i)-2 \pi i r_{2}\right) n / c\right)}{n^{2 k+2} \cosh ((\beta+\pi i) n / c)} \\
-(-1)^{\left[r_{1}+r_{2}\right]} 2^{2 k+2} \sum_{j=1}^{c}(-1)^{j} \sum_{\ell=0}^{2 k+3} \frac{B_{\ell}\left(\left(j-\left\{r_{1}+r_{2}\right\}\right) / c\right)}{\ell !} \\
. \frac{\bar{B}_{2 k+3-\ell}\left(\left(j+r_{2}-\left\{r_{1}+r_{2}\right\}\right) / c\right)}{(2 k+3-\ell) !}(-\pi i)^{\ell} \alpha^{k-\ell+3 / 2} .
\end{gathered}
$$

Proof. Let $h=(1 / 2,0)$ and let $m=2 k+1$ in $(2.1)$. In similar to (2.14), (2.15) and (2.16), we obtain that

$$
\begin{aligned}
& H(V \tau,-2 k-1 ; r, h) \\
& \quad=(-1)^{\left[r_{1}\right]} \sum_{n=1}^{\infty} \frac{\cosh \left(\pi i\left(\left(2\left\{r_{1}\right\}-1\right)(1-1 / z)+2 r_{2}\right) n / c\right)}{n^{2 k+1} \cosh (\pi i(1-1 / z) n / c)},
\end{aligned}
$$

$$
\begin{aligned}
& H(\tau,-2 k-1 ; R, H) \\
& =(-1)^{\left[r_{1}+r_{2}\right]} \sum_{n=1}^{\infty} \frac{\cosh \left(\pi i\left(\left(2\left\{r_{1}+r_{2}\right\}-1\right)(z-1)+2 r_{2}\right) n / c\right)}{n^{2 k+1} \cosh (\pi i(z-1) n / c)}
\end{aligned}
$$

and

$$
\begin{array}{r}
L(\tau,-2 k ; R, H)=(-1)^{\left[r_{1}+r_{2}\right]} 2 \pi i \sum_{j=1}^{c}(-1)^{j} \sum_{\ell=0}^{2 k+3} \frac{B_{\ell}\left(\left(j-\left\{r_{1}+r_{2}\right\}\right) / c\right)}{\ell !} \\
\cdot \frac{\bar{B}_{2 k+3-\ell}\left(\left(j+r_{2}-\left\{r_{1}+r_{2}\right\}\right) / c\right)}{(2 k+3-\ell) !}(-z)^{\ell-1} .
\end{array}
$$

Applying (2.14), (2.15) and (2.16) to (2.1), we arrive at the desired results.

If $h=(1 / 2,0), m=2 k+1$ and $z=\pi i / \alpha$ in (2.1) when $c$ is odd, then we obtain the complex conjugate of the second series identity in Theorem 2.4. If $r_{1}=1 / 2$ and $r_{2}=0$ in Theorem 2.12, then we obtain Corollary 3.10 in [4].

Theorem 2.13. Let $\alpha, \beta>0$ with $\alpha \beta=\pi^{2}$. Let $r_{1}$ and $r_{2}$ be real numbers such that $r_{1}$ and $r_{1}+r_{2}$ are not integers. Then, for any integer $k$ and for any positive integer $c$,

$$
\begin{aligned}
& \alpha^{-k} \sum_{n=0}^{\infty} \frac{\cosh \left(\left(\left(2\left\{r_{1}\right\}-1\right)(\alpha-\pi i)-2 \pi i r_{2}\right)(2 n+1) /(2 c)\right)}{(2 n+1)^{2 k+1} \sinh ((\alpha-\pi i)(2 n+1) /(2 c))} \\
& =(-\beta)^{-k} \sum_{n=0}^{\infty} \frac{\cosh \left(\left(\left(2\left\{r_{1}+r_{2}\right\}-1\right)(\beta+\pi i)-2 \pi i r_{2}\right)(2 n+1) /(2 c)\right)}{(2 n+1)^{2 k+1} \sinh ((\beta+i \pi)(2 n+1) /(2 c))} \\
& \quad-\frac{1}{4} \sum_{j=1}^{c}(-1)^{\left[\left(j+r_{2}-\left\{r_{1}+r_{2}\right\}\right) / c\right]} \sum_{\ell=0}^{2 k} \frac{E_{\ell}\left(\left(j-\left\{r_{1}+r_{2}\right\}\right) / c\right)}{\ell !}
\end{aligned}
$$




$$
\cdot \frac{\bar{E}_{2 k-\ell}\left(\left(j+r_{2}-\left\{r_{1}+r_{2}\right\}\right) / c\right)}{(2 k-\ell) !}(-\pi i)^{\ell+1} \alpha^{k-\ell} .
$$

Proof. Let $z=\pi i / \alpha, h=(0,1 / 2)$ and $m=2 k$ in (2.1). We obtain from (2.2) and (2.3) that

$$
\begin{aligned}
& H(V \tau,-2 k ; r, h) \\
& =2^{2 k+1} \sum_{n=0}^{\infty} \frac{e^{\pi i\left(\left\{r_{1}\right\}(1-1 / z)+r_{2}\right)(2 n+1) / c}+e^{\pi i\left(\left(1-\left\{r_{1}\right\}\right)(1-1 / z)-r_{2}\right)(2 n+1) / c}}{(2 n+1)^{2 k+1}(1-e((1-1 / z)(2 n+1) /(2 c)))} \\
& =2^{2 k+1} \sum_{n=0}^{\infty} \frac{\cosh \left(\pi i\left(\left(2\left\{r_{1}\right\}-1\right)(1-1 / z)+2 r_{2}\right)(2 n+1) /(2 c)\right)}{(2 n+1)^{2 k+1} \sinh (\pi i(1 / z-1)(2 n+1) /(2 c))}
\end{aligned}
$$

and

$$
\begin{aligned}
& H(\tau,-2 k ; R, H) \\
& =2^{2 k+1} \sum_{n=0}^{\infty} \frac{e^{\pi i\left(\left\{r_{1}+r_{2}\right\}(z-1)+r_{2}\right)(2 n+1) / c}+e^{\pi i\left(\left(1-\left\{r_{1}+r_{2}\right\}\right)(z-1)-r_{2}\right)(2 n+1) / c}}{(2 n+1)^{2 k+1}(1-e((z-1)(2 n+1) /(2 c)))} \\
(2.21) \quad= & 2^{2 k+1} \sum_{n=0}^{\infty} \frac{\cosh \left(\pi i\left(\left(2\left\{r_{1}+r_{2}\right\}-1\right)(z-1)+2 r_{2}\right)(2 n+1) /(2 c)\right)}{(2 n+1)^{2 k+1} \sinh (\pi i(1-z)(2 n+1) /(2 c))} .
\end{aligned}
$$

Since $H_{1} \equiv H_{2} \equiv 1 / 2(\bmod 1)$,

$$
\begin{aligned}
& L(\tau,-2 k ; R, H)= \sum_{j=1}^{c} e\left(-\frac{1}{2}\left[\frac{j+r_{2}-\left\{r_{1}+r_{2}\right\}}{c}\right]\right) \\
& \cdot \int_{C} u^{-2 k-1} \sum_{m=0}^{\infty} \bar{E}_{m}\left(\frac{j+\varrho}{c}\right) \frac{u^{m}}{m !} \sum_{n=0}^{\infty} E_{n}\left(\frac{j-\left\{R_{1}\right\}}{c}\right) \frac{(-z u)^{n}}{n !} \\
&= \frac{1}{2} \pi i \sum_{j=1}^{c}(-1)^{\left[\left(j+r_{2}-\left\{r_{1}+r_{2}\right\}\right) / c\right]} \sum_{\ell=0}^{2 k} \frac{E_{\ell}\left(\left(j-\left\{r_{1}+r_{2}\right\}\right) / c\right)}{\ell !} \\
& \cdot \frac{\bar{E}_{2 k-\ell}\left(\left(j+r_{2}-\left\{r_{1}+r_{2}\right\}\right) / c\right)}{(2 k-\ell) !}(-z)^{\ell} .
\end{aligned}
$$

Put $z=\pi i / \alpha$ and apply (2.20), (2.21) and (2.22) to (2.1). Then we deduce Theorem 2.13 .

Corollary 2.14. For any positive integer $c$,

$$
\begin{aligned}
\alpha^{-k} & \sum_{n=0}^{\infty} \frac{\operatorname{csch}((\alpha-\pi i)(2 n+1) /(2 c))}{(2 n+1)^{2 k+1}} \\
& =(-\beta)^{-k} \sum_{n=0}^{\infty} \frac{\operatorname{csch}((\beta+\pi i)(2 n+1) /(2 c))}{(2 n+1)^{2 k+1}} \\
& -\frac{1}{4} \sum_{j=1}^{c} \sum_{\ell=0}^{2 k} \frac{E_{\ell}((j-1 / 2) / c) E_{2 k-\ell}((j-1 / 2) / c)}{\ell !(2 k-\ell) !}(-\pi i)^{\ell+1} \alpha^{k-\ell} .
\end{aligned}
$$

Proof. Put $r_{1}=1 / 2$ and $r_{2}=0$ in Theorem 2.13. 
Corollary 2.14 should be compared with Corollary 3.3 in [4].

Corollary 2.15 .

$$
\begin{aligned}
\alpha^{-k} \sum_{n=0}^{\infty} \frac{(-1)^{n} \operatorname{sech}((2 n+1) \alpha / 2)}{(2 n+1)^{2 k+1}} & =-(-\beta)^{-k} \sum_{n=0}^{\infty} \frac{(-1)^{n} \operatorname{sech}((2 n+1) \beta / 2)}{(2 n+1)^{2 k+1}} \\
& +\frac{\pi}{4} \sum_{\ell=0}^{k} \frac{E_{2 \ell}(1 / 2) E_{2 k-2 \ell}(1 / 2)}{(2 \ell) !(2 k-2 \ell) !} \alpha^{k-\ell}(-\beta)^{\ell} .
\end{aligned}
$$

0 .

Proof. Put $c=1$ in Corollary 2.14 and apply $E_{2 n+1}\left(\frac{1}{2}\right)=0, n \geq$

Corollary 2.15 has been stated in Ramanujan's Notebook [6].

Corollary 2.16. For any positive integer $M$,

$$
\alpha^{2 M-1} \sum_{n=0}^{\infty} \frac{(-1)^{n} \operatorname{sech}((2 n+1) \alpha / 2)}{(2 n+1)^{-4 M+3}}=\beta^{2 M-1} \sum_{n=0}^{\infty} \frac{(-1)^{n} \operatorname{sech}((2 n+1) \beta / 2)}{(2 n+1)^{-4 M+3}} \text {. }
$$
0 .

Proof. Put $c=1$ in Corollary 2.14 and let $k=-2 M+1$ for $M>$

Theorem 2.17. Let $\alpha, \beta>0$ with $\alpha \beta=\pi^{2}$. Let $r_{1}$ and $r_{2}$ be real numbers such that $r_{1}$ and $r_{1}+r_{2}$ are not integers. Then, for any integer $k$ and for any positive integer $c$,

$$
\begin{gathered}
\alpha^{-k-1 / 2} \sum_{n=0}^{\infty} \frac{\sinh \left(\left(\left(2\left\{r_{1}\right\}-1\right)(\alpha-\pi i)-2 \pi i r_{2}\right)(2 n+1) /(2 c)\right)}{(2 n+1)^{2 k+2} \sinh ((\alpha-\pi i)(2 n+1) /(2 c))} \\
=(-\beta)^{-k-1 / 2} \sum_{n=0}^{\infty} \frac{\sinh \left(\left(\left(2\left\{r_{1}+r_{2}\right\}-1\right)(\beta+\pi i)-2 \pi i r_{2}\right)(2 n+1) /(2 c)\right)}{(2 n+1)^{2 k+2} \sinh ((\beta+i \pi)(2 n+1) /(2 c))} \\
+\frac{1}{4} \sum_{j=1}^{c}(-1)^{\left[\left(j+r_{2}-\left\{r_{1}+r_{2}\right\}\right) / c\right]} \sum_{\ell=0}^{2 k+1} \frac{E_{\ell}\left(\left(j-\left\{r_{1}+r_{2}\right\}\right) / c\right)}{\ell !} \\
\cdot \frac{\bar{E}_{2 k+1-\ell}\left(\left(j+r_{2}-\left\{r_{1}+r_{2}\right\}\right) / c\right)}{(2 k+1-\ell) !}(-\pi i)^{\ell+1} \alpha^{k-\ell+1 / 2} .
\end{gathered}
$$

Proof. Let $h=(0,1 / 2)$ and $m=2 k+1$ in $(2.1)$. By the same matter in $(2.20),(2.21)$ and $(2.22)$, we have

$$
\begin{aligned}
& H(V \tau,-2 k-1 ; r, h) \\
& \quad=2^{2 k+2} \sum_{n=0}^{\infty} \frac{\sinh \left(\pi i\left(\left(2\left\{r_{1}\right\}-1\right)(1-1 / z)+2 r_{2}\right)(2 n+1) /(2 c)\right)}{(2 n+1)^{2 k+2} \sinh (\pi i(1 / z-1)(2 n+1) /(2 c))}, \\
& H(\tau,-2 k-1 ; R, H) \\
& \quad=2^{2 k+2} \sum_{n=0}^{\infty} \frac{\sinh \left(\pi i\left(\left(2\left\{r_{1}+r_{2}\right\}-1\right)(z-1)+2 r_{2}\right)(2 n+1) /(2 c)\right)}{(2 n+1)^{2 k+2} \sinh (\pi i(1-z)(2 n+1) /(2 c))}
\end{aligned}
$$


and

$$
\begin{gathered}
L(\tau,-2 k-1 ; R, H)=\frac{1}{2} \pi i \sum_{j=1}^{c}(-1)^{\left[\left(j+r_{2}-\left\{r_{1}+r_{2}\right\}\right) / c\right]} \sum_{\ell=0}^{2 k+1} \frac{E_{\ell}\left(\left(j-\left\{r_{1}+r_{2}\right\}\right) / c\right)}{\ell !} \\
. \frac{\bar{E}_{2 k+1-\ell}\left(\left(j+r_{2}-\left\{r_{1}+r_{2}\right\}\right) / c\right)}{(2 k+1-\ell) !}(-z)^{\ell} .
\end{gathered}
$$

Take $z=\pi i / \alpha$ and plug (2.23), (2.24) and (2.25) into (2.1). Then the desired results follow.

Corollary 2.18.

$$
\begin{aligned}
& \alpha^{-k-1 / 2} \sum_{n=0}^{\infty} \frac{\operatorname{sech}((\alpha-\pi i)(2 n+1) /(4 c))}{(2 n+1)^{2 k+2}} \\
& =(-\beta)^{-k-1 / 2} \sum_{n=0}^{\infty} \frac{\operatorname{sech}((\beta+\pi i)(2 n+1) /(4 c))}{(2 n+1)^{2 k+2}} \\
& \quad-\frac{1}{4} \sum_{j=1}^{c} \sum_{\ell=0}^{2 k+1} \frac{E_{\ell}((j-1 / 4) / c)}{\ell !} \frac{\bar{E}_{2 k+1-\ell}((j-1 / 4) / c)}{(2 k+1-\ell) !}(-\pi i)^{\ell+1} \alpha^{k-\ell+1 / 2} .
\end{aligned}
$$

Proof. Let $r_{1}=1 / 4$ and $r_{2}=0$ in Theorem 2.17

Corollary 2.18 should be compared with Corollary 3.10 in [4].

Corollary 2.19.

$$
\begin{aligned}
\alpha^{1 / 2} \sum_{n=0}^{\infty} \operatorname{sech} & ((\alpha-\pi i)(2 n+1) /(4 c)) \\
= & (-\beta)^{1 / 2} \sum_{n=0}^{\infty} \operatorname{sech}((\beta+\pi i)(2 n+1) /(4 c)) .
\end{aligned}
$$

Proof. Let $k=-1$ in Corollary 2.18.

\section{References}

[1] B.C. Berndt, Two new proofs of Lerch's functional equation, Proc. Amer. Math. Soc. 32 (1972), 403-408.

[2] B.C. Berndt, Modular transformations and generalizations of several formulae of Ramanujan, Rocky Mountain J. Math. 7 (1) (1977), 147-189.

[3] B.C. Berndt, Analytic Eisenstein series, theta-functions, and series relations in the spirit of Ramanujan, J. Reine Angew. Math. 304 (1978), 332-365.

[4] S. Lim, Infinite series Identities from modular transformation formulas that stem from generalized Eisenstein series, Acta Arith. 141 (3) (2010), 241-273.

[5] S. Lim, Series relations from certain modular transformation formula, J. Chungcheong Math. Soc. 24 (3) (2011), 481-502.

[6] S. Ramanujan, Notebooks of Srinivasa Ramanujan (2 volumes), Tata Institute of Fundamental Research, Bombay, 1957. 
Department of Mathematics

Mokwon University

Mokwon Gil 21, Seo-gu, Daejeon

302-318, Republic of Korea

E-mail: sglimj@mokwon.ac.kr 